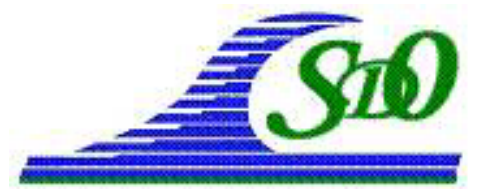

Xìmes Journées Nationales Génie Côtier - Génie Civil

Les Sables d'Olonne, 22-25 juin 2010

DOI:10.5150/jngcgc.2010.039-HＣ Editions Paralia CFL

disponible en ligne - http://www.paralia.fr - available online

\title{
Climat de houle et caractéristiques morpho-sédimentaires du littoral de Bouknadel-Kénitra (Maroc)
}

\author{
Mounir HAKKOU ${ }^{1}$, Bruno CASTELLE ${ }^{2}$, \\ Aïcha BENMOHAMMADI ${ }^{1}$, Bendahhou ZOURARA ${ }^{3}$
}

1. Département de géologie, faculté des sciences, université Ibn-Tofaïl, B.P. 133, Kénitra, Maroc.

monhak@yahoo.fr

2. CNRS, UMR EPOC 5805, Talence, F-33405, France.

b.castelle@epoc.u-bordeauxl.fr

3. Laboratoire de Géosciences Marines, Département de Géologie, Faculté des Sciences, B.P. 20, El Jadida, Maroc.

\section{Résumé :}

La côte de Bouknadel-Kenitra (Maroc) est constituée de plages sableuses énergétiques quasiment rectilignes qui s'étendent sur environ $20 \mathrm{~km}$ de linéaire côtier avec un marnage méso-tidal. Les plages sableuses sont interrompues au Nord par l'embouchure du Sebou fixée par deux digues s'étendant sur environ $600 \mathrm{~m}$ vers le large. Le littoral encourt actuellement un risque sérieux de dégradation sous l'effet des nouvelles perspectives de son occupation touristique et résidentielle susceptibles de déséquilibrer ses unités morphologiques fragiles. La dynamique hydro-sédimentaire de ce littoral est à ce jour très peu documentée et les données sont quasi-inexistantes. S'inscrivant dans ce contexte, ce papier apporte un premier aperçu de son comportement morphosédimentaire afin d'aider à la décision de son aménagement. L'approche combine un suivi biannuel de profils de plage le long de ce littoral, l'analyse granulométrique des sables relevés le long des profils, les données sur 9 ans du modèle de vagues global WW3 et d'une modélisation empirique des conditions de houle au déferlement et du transit sédimentaire généré. Une quantification des transits sédimentaires et une classification de la morphologie des plages ont été réalisées et confrontées aux observations de terrain, permettant ainsi de déduire de manière plus générale des conclusions intéressantes sur le comportement des plages sableuses soumises à l'action de la houle à proximité des ouvrages côtiers.

\section{Mots-clés :}

Dérive littorale - Classification des plages - Profil de plage - Aménagements côtiers Climat de houle

\section{Introduction}

Le littoral de Bouknadel-Kenitra, qui appartient au plateau continental Nord Atlantique marocain, comporte un ensemble de plages sableuses bordées par de hautes dunes qui 
s'étirent sur environ $20 \mathrm{~km}$ de linéaire côtier (figure 1). Au nord, on distingue la présence de l'embouchure de Sebou prolongée par deux digues s'étendant jusqu'à 600 $\mathrm{m}$ au large, bloquant ainsi les transits sédimentaires. La marée est semi-diurne avec un marnage de type méso-tidal. La côte est exposée aux houles énergétiques générées dans l'Atlantique Nord qui peuvent fréquemment dépasser les $5 \mathrm{~m}$ en période hivernale. Des images satellites et aériennes révèlent des signes de déséquilibre sédimentaire de part et d'autre du Sebou et des problèmes d'érosion préoccupant sont observés sur plusieurs zones. Malgré cela, un grand nombre de projets immobiliers et touristiques sont en cours renforçant la menace qui pèse sur ce littoral. Ce papier vise à mieux comprendre et quantifier les forçages hydro-sédimentaires le long de ces plages.

\section{Matériel et méthode}

\subsection{Suivi de profils, analyse granulométrique et classification des plages}

Dans ce papier nous présentons un suivi biannuel de profils de plage le long du secteur d'étude et une analyse granulométrique des sables relevés le long de ces profils en bas, en haut et au milieu de l'estran. Comme on peut le voir sur les figures $1 \mathrm{~b}, 1 \mathrm{c}$ et $1 \mathrm{~d}$, la morphologie des plages présente une importante variabilité le long de la côte. Afin de comprendre cette variabilité, 12 profils de référence ont été définis le long du secteur de Bouknadel-Kénitra (figure 1a). A partir de ces données une classification des états de plage peut être générée le long du littoral à partir du modèle conceptuel de WRIGHT \& SHORT (1984) fondé sur l'indice de DEAN (1973) $\Omega=H b / W s T$ où $H b$ est la hauteur des vagues au déferlement, $T$ la période des vagues et $W_{S}$ la vitesse de chute du sédiment.

\subsection{Modélisation : vagues et transit littoral}

Pour appréhender la climatologie de houle nous avons téléchargé les données de sortie du modèle de vagues globale WW3 (TOLMAN, 1991) toutes les 3 heures de janvier 1998 à décembre 2006: la hauteur significative $H s$, la période pic $T p$ et la direction de propagation $\alpha_{p}$ pour le nœud de WW3 le plus proche de notre secteur (N3400'00 $\mathrm{E} 7^{\circ} 15^{\prime} 00$ ), situé à $32 \mathrm{~km}$ du rivage. Ces données ont été utilisées pour décrire la climatologie de houle mais aussi pour ensuite estimer empiriquement la dérive littorale et ses variabilités annuelles, inter-annuelles et spatiales le long de ce littoral.

Afin d'estimer la quantité de sable transporté le long de la plage, nous nous sommes basés sur la formulation proposée par KACZMAREK et al. (2005). Cette formule a été choisie en raison de son nombre limité des paramètres ajustables et aussi parce qu'elle a été utilisée et validée par BERTIN et al. (2008) et CASTELLE et al. (2009) dans des environnements hydro-sédimentaires similaires à ceux de notre zone d'étude. Cette formule s'écrit : 

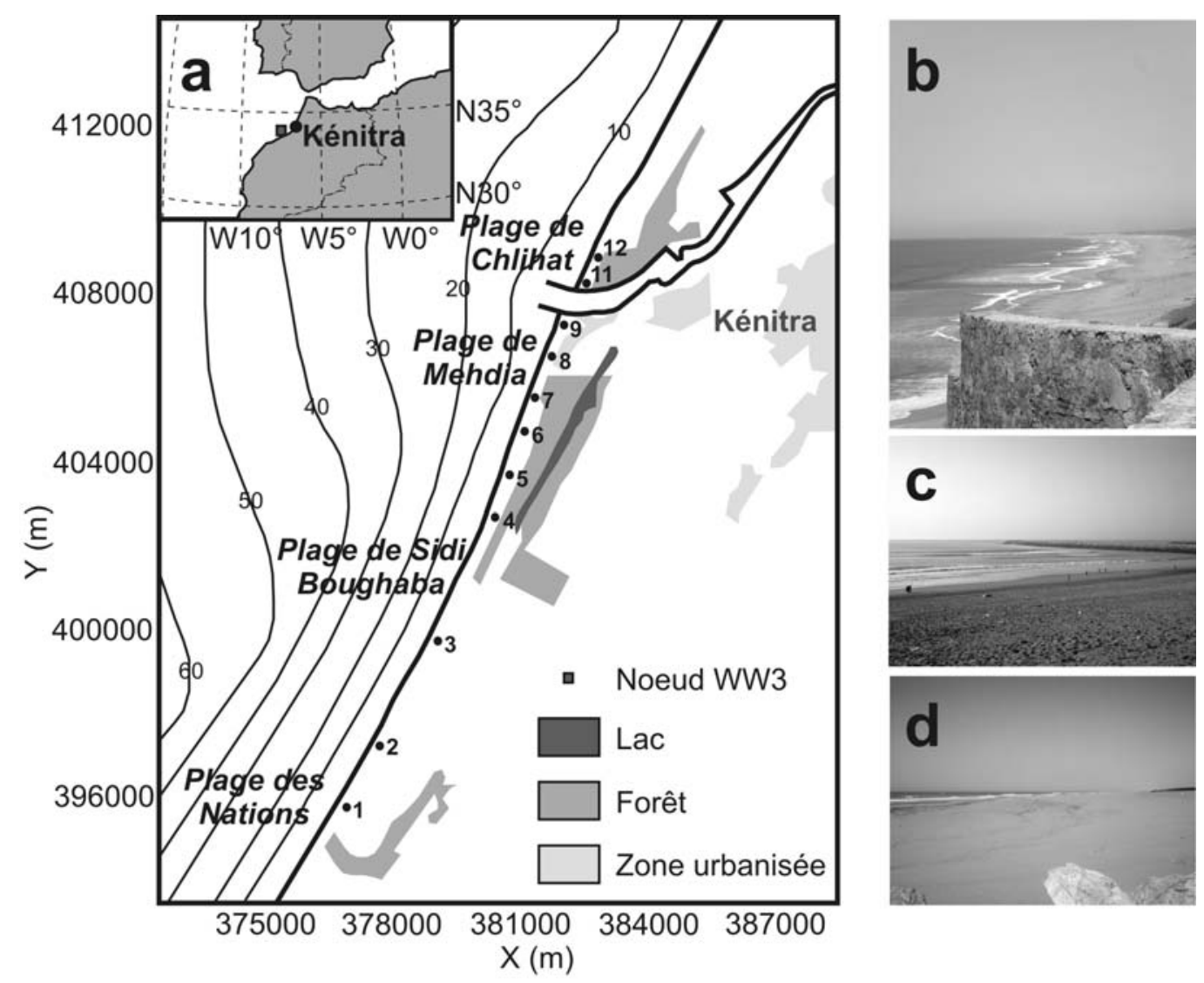

Figure 1. Plages de Bouknadel-Kénitra avec (a) leur description géographique et positionnement des profils de plage mesurés dans cette étude. Sont présentées également les photos prises de (b) la plage des Nations, (c) la plage de Medhia et (d) la plage de Chlihat qui montrent la forte variabilité des morphologies observées le long du secteur de Bouknadel-Kénitra.

$Q=0,023 \cdot\left(H_{b}^{2} \cdot V\right)$ si $\left(\mathrm{H}_{\mathrm{b}}^{2} . \mathrm{V}\right)<0,15$

$Q=0,00225+0,008 \cdot\left(H_{b}^{2} \cdot V\right) \quad$ si $\left(H_{b}^{2} \cdot V\right)>0,15$

où $H b$ est la hauteur des vagues au déferlement et $V$ une mesure du courant de dérive donnée par:

$V=0,25 \cdot k_{v} \cdot \sqrt{\gamma \cdot g \cdot H_{b}} \cdot \sin 2 \alpha$

où $\alpha$ est l'angle d'incidence des vagues au point de déferlement, $\gamma$ est l'indice de déferlement de BATTJES \& JANSSEN (1978), $k_{v}$ une constante empirique fixée à 2,9 (BERTIN et al., 2008; CASTELLE et al., 2009). Les conditions au point de déferlement $\left(H_{b}, \alpha\right)$ sont déterminées à partir de la loi de Snell-Descartes combinée à la formule de RATTANAPITIKON \& SHIBAYANA (2000). Cette démarche considère des isobathes régulières et parallèles au rivage. Des résultats préliminaires réalisés avec le modèle SWAN (BOOIJ et al., 1999) montrent que cette hypothèse d'uniformité des isobathes est valable pour tout le secteur d'étude sauf au niveau de la plage de Mehdia qui concentre considérablement les houles dominantes du N-O. 


\section{Résultats}

\subsection{Climatologie de houle}

L'analyse des données WW3 montre que la côte de Bouknadel-Kenitra est principalement exposée aux houles du secteur $\mathrm{O}-\mathrm{NO}$, de périodes typiquement comprises entre 10 et $15 \mathrm{~s}$, qui sont générées par les dépressions traversant l'Atlantique Nord. $70 \%$ des houles ont une hauteur significative comprise entre 0,5 et $2 \mathrm{~m}$ avec une incidence centrée autour de la direction NO (figure 2). Par conséquent, ces houles tendent à venir attaquer la côte avec une incidence quasi-normale à la côte suggérant dès à présent une dérive littorale nette relativement faible par rapport aux conditions relativement énergétiques. Il est à noter que pendant la période estivale la côte est exposée à des mers de vent peu énergétiques de N-NE générées localement par les alizées (figure 2).
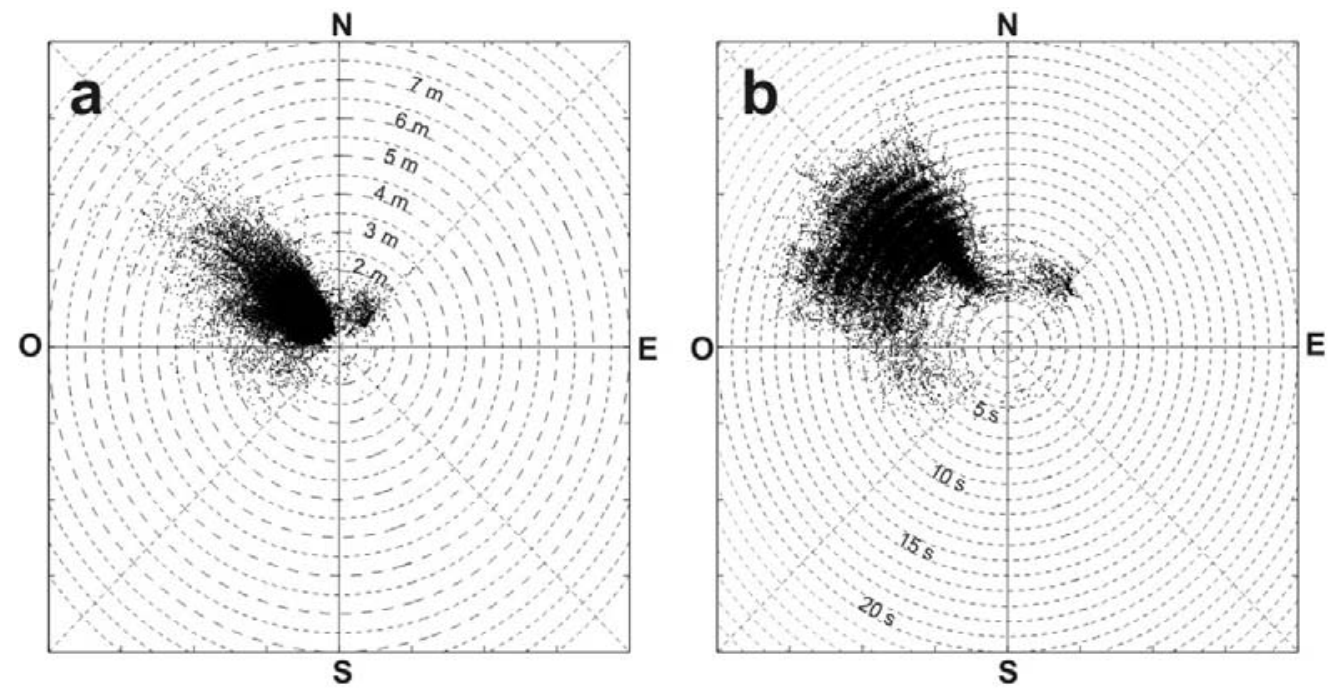

Figure 2. Caractérisation des vagues au noud WW3 avec (a) Hs en fonction de l'angle d'incidence et (b) Tp en fonction de l'angle d'incidence.

\subsection{Morphologie des plages de Bouknadel-Kénitra}

L'observation sur le terrain montre une forte variabilité de la morphologie des plages le long de cette section du littoral marocain. On note notamment souvent l'omniprésence de systèmes barre/chenal au niveau de la Plage des Nations (figure 1b) qui contraste avec un haut de plage raide et des bancs de sable au large complexes à Medhia (figure 1c) et un profil très dissipatif au nord du Sébou à Chlihat (figure 1d). Ces observations sont confirmées sur la figure 3 qui montre les profils typiques d'hiver et d'été mesurés pendant notre étude le long de 11 profils représentatifs de la variabilité de ces plages (figure 1a, le profil 10 n'est pas utilisé car le point de référence à été perdu lors d'une tempête). 

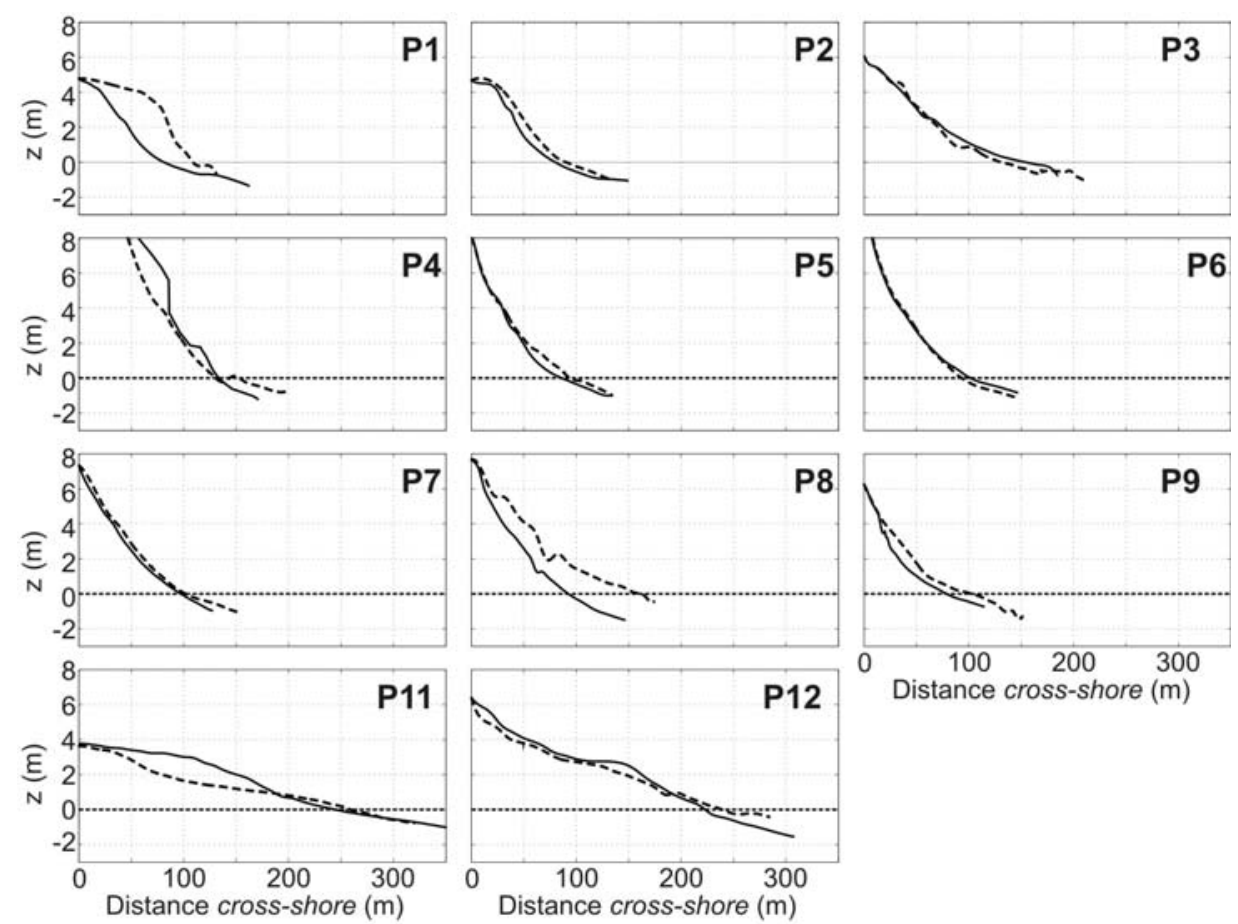

Figure 3. Profils de plage mesurés le long des points de référence donnés en figure la en aout 2008 (pointillés) et janvier 2009 (trait plein). La cote 0 correspond au niveau moyen des mers.

Ces relevés confirment les observations récurrentes réalisées le long de ces plages et mettent en avant de fortes variabilités des pentes. On remarque également des profils assez semblables entre l'été et l'hiver avec globalement une érosion assez faible du littoral.

Nous avons réalisé une classification des plages à partir des données de WW3 et des données sédimentaires prélevées le long des profils. Ces données sont synthétisées dans le tableau 1. Les résultats montrent que, sans surprise, les plages du secteur BouknadelKénitra appartiennent aux plages intermédiaires $(1<\Omega<6)$. Ils montrent aussi qu'à partir du nombre de Dean, les plages sont globalement de plus en plus dissipatives en se déplaçant vers le nord, ce qui est en accord avec les observations de terrain. Toutefois, ces différences sont faibles par rapport à certaines morphologies très contrastées qu'on observe fréquemment comme dans le cas de la plage des Nations et de Chlihat (figures $5 \mathrm{~b}$ et $5 \mathrm{~d}$, ou entre profils P1 et P11 sur la figure 3 ).

\subsection{Dérive littorale}

Le long de ce secteur d'étude, la dérive littorale calculée par notre méthode est dirigée vers le sud avec une variabilité spatiale significative (tableau 2). La dérive nette vers le sud est relativement faible au niveau de Chlihat $\left(84000 \mathrm{~m}^{3} / \mathrm{an}\right)$ et est la plus importante du côté de Sidi Boughaba (338000 $\left.\mathrm{m}^{3} / \mathrm{an}\right)$. Ces valeurs de dérive littorale moyenne nette 
Thème 2 - Dynamique sédimentaire et transports des particules

vers le sud sont faibles si on considère l'énergie des vagues incidentes. Elles sont dues d'une part au fait que l'angle d'incidence à la côte est assez faible et d'autre part parce que les composantes vers le nord et vers le sud sont du même ordre de grandeur.

Tableau 1. Synthèse des données sur les profils de plage avec Hb et T respectivement la hauteur moyenne et la période moyenne des vagues pendant la saison, $d_{50}(\mu \mathrm{m})$ la taille du sédiment moyennée de long de l'estran et $W \mathrm{~s}(\mathrm{~m} / \mathrm{s})$ la vitesse de chute du sédiment.

\begin{tabular}{|c|c|c|c|c|c|c|c|c|}
\hline & \multicolumn{4}{|c|}{ Hiver $(H b=2,39 \mathrm{~m}, \mathrm{~T}=12,48 \mathrm{~s})$} & \multicolumn{4}{|c|}{ Eté $(H b=1,12 m, T=8,05 \mathrm{~s})$} \\
\hline & Pente(\%) & $d_{50}(\mu m)$ & $W s(m / s)$ & $\mathbf{\Omega}$ & Pente(\%) & $d_{50}(\mu m)$ & $W s(m / s)$ & $\mathbf{\Omega}$ \\
\hline$P 1$ & 1,49 & 368 & 0,0543 & 3,53 & 2,12 & 358 & 0,0528 & 2,64 \\
\hline$P 2$ & 1,64 & 297 & 0,0430 & 4,47 & 2,18 & 299 & 0,0433 & 3,21 \\
\hline P3 & 1,47 & 296 & 0,0428 & 4,49 & 1,26 & 307 & 0,0447 & 3,11 \\
\hline P4 & 2,69 & 322 & 0,0471 & 4,08 & 1,72 & 333 & 0,0489 & 2,85 \\
\hline P5 & 2,10 & 285 & 0,0409 & 4,69 & 2,12 & 312 & 0,0455 & 3,06 \\
\hline P6 & 1,89 & 308 & 0,0448 & 4,29 & 1,95 & 321 & 0,0470 & 2,96 \\
\hline P7 & 2,35 & 298 & 0,0431 & 4,45 & 2,00 & 323 & 0,0473 & 2,94 \\
\hline P8 & 1,83 & 305 & 0,0443 & 4,33 & 1,78 & 317 & 0,0463 & 3,00 \\
\hline P9 & 2,05 & 313 & 0,0457 & 4,21 & 1,72 & 319 & 0,0466 & 2,98 \\
\hline P10 & 0,79 & 300 & 0,0435 & 4,42 & 0,69 & 319 & 0,0466 & 2,98 \\
\hline P11 & 1,29 & 274 & 0,0390 & 4,92 & 1,02 & 301 & 0,0437 & 3,19 \\
\hline
\end{tabular}

Tableau 2. Composantes (vers le sud, vers le nord et net) de la dérive littorale moyennée sur la période 1998-2006.

\begin{tabular}{llll}
\hline Dérive littorale $\left(\mathrm{m}^{3} / \mathrm{an}\right)$ & Vers le sud & Vers le nord & Nette \\
\hline Plage de Chlihat & 310000 & 226000 & 84000 \\
Plage de Sidi Boughaba & 457000 & 119000 & 338000 \\
Plage des Nations & 371000 & 172000 & 199000 \\
\hline
\end{tabular}

Les valeurs présentées dans le tableau 2 présentent de fortes variabilités annuelles et inter-annuelles. Ces variabilités importantes sont données à titre d'exemple pour la plage de Chlihat sur la figure 4. On remarque notamment que la dérive nette annuelle peut être négligeable (en 2001 sur la figure 4) ou dépasser $350000 \mathrm{~m}^{3} /$ an (en 1999 sur la figure 4). Au niveau saisonnier, on remarque que la dérive littorale vers le nord et vers le sud est intense entre octobre et avril, période pendant laquelle les composantes s'annulent quasiment. De manière surprenante, la faible contribution vers le sud de mai à septembre est en fait responsable de la dérive net annuelle puisqu'elle n'est compensée par aucune (ou très peu) de dérive littorale vers le nord. En effet pendant cette période les houles sont beaucoup moins énergétiques mais ont aussi une orientation plus N-NO puisque les dépressions en Atlantique nord ont une trajectoire 
beaucoup plus nord. D'autre part les houles courtes générées par les alizées transportent aussi une quantité significative de sable vers le sud.

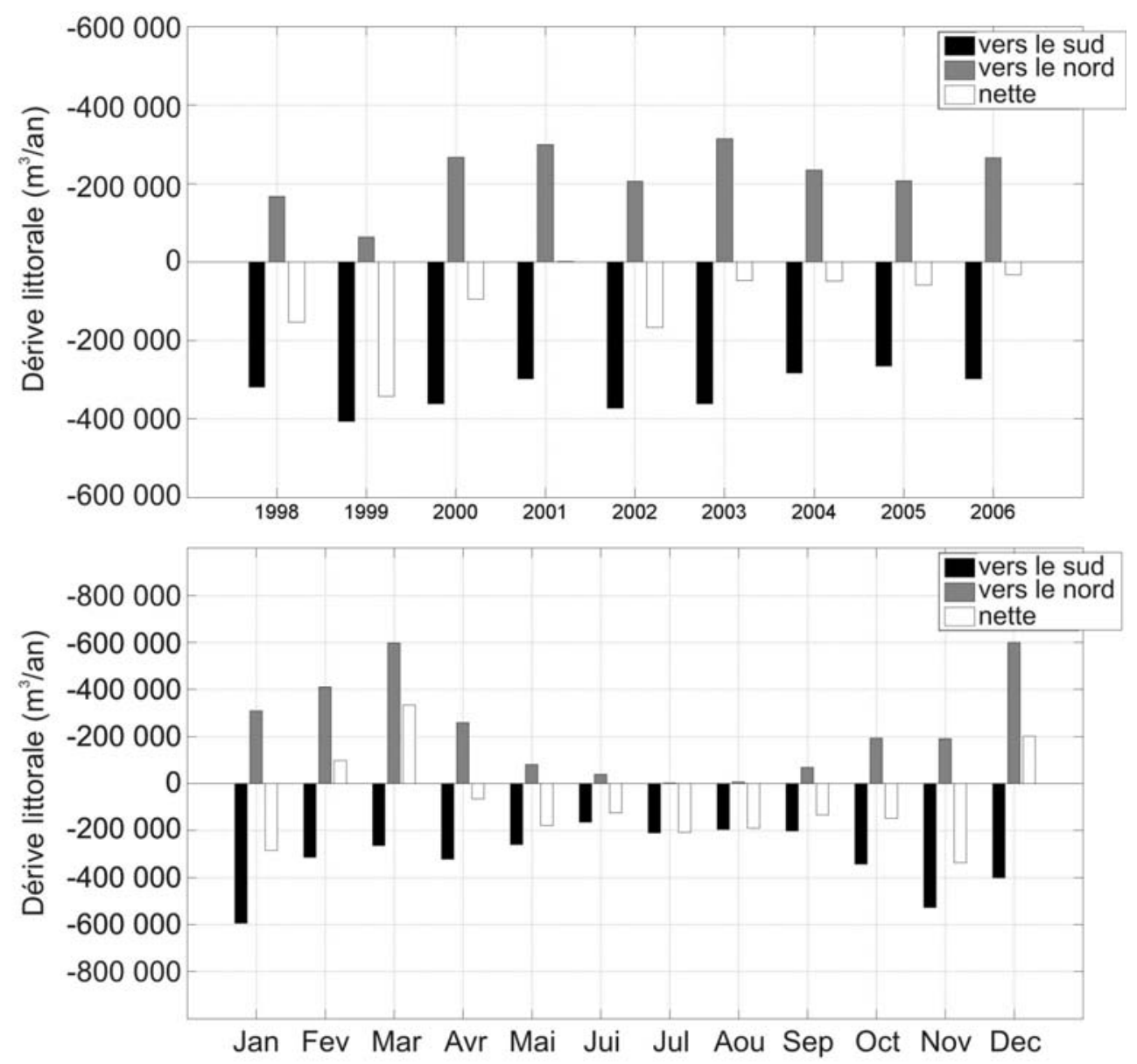

Figure 4. Variabilités temporelles des composantes (vers le sud, vers le nord et nette) de la dérive littorale à Chlihat avec en haut la variabilité inter-annuelle et en bas la variabilité annuelle.

\section{Discussion et conclusions}

L'exploitation des données WW3 combinée à la modélisation empirique de la dérive littorale a permis une première description quantitative de la climatologie de houle le long de cette section du littoral marocain ainsi que de la dérive littorale et de ses variabilités annuelles, inter-annuelles et spatiales. A noter qu'à Chlihat, les faibles valeurs de dérive nette vers le sud sont confirmées par une analyse de photographies aériennes entre 1969 et 2002 (non présentée ici) montrant un avancement de la plage de Chlihat de l'ordre $1-1,2 \mathrm{~m} / \mathrm{an}$, valeurs bien inférieures à celles observées sur la Gold Coast en Australie a proximité d'une digue similaire et pour une dérive littorale en moyenne de $500000 \mathrm{~m}^{3} / \mathrm{an}$. Cette étude, dont une partie vient d'être présentée dans ce 
papier, la première extensive sur la zone, était nécessaire notamment dans le cadre des nombreux projets immobiliers qui peuvent exploiter la dune blanche.

D'un point de vue plus général, dans ce papier nous montrons que ce type de méthode peut être appliqué à des zones peu documentées où les données sont quasi-inexistantes. Elle doit être toutefois combinée avec des mesures de terrain et des analyses sédimentaires afin de déterminer les caractéristiques morpho-sédimentaires d'un littoral sableux. Enfin nous avons montré que les états de plages prédits par l'indice de Dean $\Omega$, peuvent être significativement différents de ceux observés sur le terrain, particulièrement à proximité des digues du Sébou. ANTHONY (1998) ou JACKSON et al. (2005) avaient déjà observé ce type de lacune et l'avaient attribué aux contrôles géologiques locaux. De manière similaire ici, nous pensons que les aménagements côtiers impactent considérablement la classification des plages.

\section{Références bibliographiques}

ANTHONY E.J. (1998). Sediment-wave parametric characterization of beaches. J. of Coast. Res., 14, 347-352.

BERTIN X., CASTELlE B., CHAUMILLON E., BUTEL R., QUIQUE R. (2008). Longshore drift estimation and inter-annual variability at a high-energy dissipative beach: St. Trojan Beach, SW Oléron Island, France. C. Shelf Res., 28, pp 1316-1332. doi:10.1016/j.csr.2008.03.005

BOOIJ N., RIS R.C., HOLTHUIJSEN L. H. (1999). A third-generation wave model for coastal regions, part I: model description and validation. J. Geophys. Res., 104(C4), pp 7649-7666. doi:10.1029/98JC02622

CASTELLE B, TURNER I.L., BERTIN X., TOMLINSON R.B. (2009). Beach nourishments at Coolangatta Bay over the period 1987-2005 : impacts and lessons. Coast. Eng., 56, pp 940-950. doi:10.1016/j.coastaleng.2009.05.005

DEAN R.G. (1973). Heuristic models of sand transport in the surf zone. Proc. $1^{\text {st }}$ Aust. Conf. on Coast. Eng., Australia, pp 208-214.

JACKSON D.W.T., COOPER J.A.G., DEL RIO L. (2005). Geological control of beach morphodynamic state. Mar. Geol., 216, pp 297-314. doi:10.1016/j.margeo.2005.02.021

KACZMAREK J.M., OSTROWSKI R, PRUSZAK Z., ROZYNSKI G. (2005). Selected problems of sediment transport and morphodynamics of a multi-bar nearshore zone. Estuar., Coast. and Shelf Sci, 62, pp 415-425. doi:10.1016/j.ecss.2004.09.006

TOLMAN H.L. (1991). A third-generation model for wind waves on slowly varying, unsteady and inhomogeneous depths and currents. J. Phys. Oceanogr, 21, pp 782-797. doi:10.1175/1520-0485(1991)021<0782:ATGMFW >2.0.CO;2

WRIGHT L.D., SHORT A.D. (1984). Morphodynamic variability of surf zones and beaches: a synthesis. Mar. Geol., 56, pp 93-118. doi:10.1016/0025-3227(84)90008-2 